\title{
Sharp bounds on the bias of trimean
}

\author{
Mariusz Bieniek
}

Received: 16 June 2014 / Revised: 4 October 2014 / Published online: 31 December 2014

(C) The Author(s) 2014. This article is published with open access at Springerlink.com

\begin{abstract}
We derive optimal bounds on the bias of approximation of unknown mean of the parent population by Tukey's trimean defined as the weighted average of the sample median and sample quartiles. The bounds are expressed in standard deviation units and the distributions for which the bounds are attained are specified. The results are illustrated with numerical example.
\end{abstract}

Keywords Bias $\cdot$ Trimean $\cdot L$-statistic $\cdot$ Schwarz' inequality

Mathematics Subject Classification $\quad 62 \mathrm{G} 30 \cdot 60 \mathrm{E} 15$

\section{Introduction}

Consider the random sample $X_{1}, \ldots, X_{n}$ with common cumulative distribution function (cdf) $F$ and the quantile function $F^{-1}(u)=\sup \{x \in \mathbb{R}: F(x) \leq u\}$ for $u \in[0,1]$. Assume that the mean $\mu=E X_{1}$ and the variance $\sigma^{2}=\operatorname{Var} X_{1}$ of the parent population are finite, so that

$$
\mu=\int_{0}^{1} F^{-1}(u) \mathrm{d} u,
$$

and

$$
\sigma^{2}=\int_{0}^{1}\left(F^{-1}(u)-\mu\right)^{2} \mathrm{~d} u .
$$

M. Bieniek $(\varangle)$

Institute of Mathematics, University of Maria Curie-Skłodowska, pl. M. Curie Skłodowskiej 1, 20-031 Lublin, Poland

e-mail: mariusz.bieniek@umcs.lublin.pl 
Let $X_{1: n} \leq \ldots \leq X_{n: n}$ denote the order statistics of the sample $X_{1}, \ldots, X_{n}$. We consider the problem of estimation of unknown $\mu$ by $L$-statistics, i.e. linear combinations of order statistics. Rychlik (1998) provided $p$-norm upper bounds on expectations of $L$-statistics and Goroncy (2009) considered lower bounds on positive $L$-statistics. In particular, Danielak and Rychlik (2003) considered single order statistics and trimmed means, and their results were strengthened by Danielak (2003) for distributions with decreasing density or failure rate. Raqab (2007) considered left-sided Winsorized means and Bieniek (2014a) extended his results to two-sided Winsorized means. In the context of generalized order statistics, optimal bounds on expectations of arbitrary $L$-statistic from bounded populations were derived by Rychlik (2010). Recently Bieniek (2014b) provided bounds on the bias of quasimidranges

$$
M_{r, s}=\frac{1}{2}\left(X_{r: n}+X_{s: n}\right), \quad 1 \leq r<s \leq n,
$$

i.e. arithmetic means of two fixed order statistics.

In this paper we consider another $L$-statistic, namely the sample trimean $T_{n}$ introduced by Tukey (1977) as an element of a set of statistical techniques in descriptive statistics called "exploratory data analysis". The trimean $T_{n}$ is defined as

$$
T_{n}=\frac{1}{4}\left(H_{1}+2 M+H_{2}\right),
$$

where $M$ is the sample median and $H_{1}$ and $H_{2}$ are lower and upper hinges of the sample. The sample median is defined usually as

$$
M= \begin{cases}X_{\frac{n+1}{2}: n}, & \text { if } n \text { is odd } \\ \frac{1}{2}\left(X_{\frac{n}{2}: n}+X_{\frac{n}{2}+1: n}\right), & \text { if } n \text { is even }\end{cases}
$$

which can be written in a more compact way as

$$
M=\frac{1}{2}\left(X_{\left\lfloor\frac{n+1}{2}\right\rfloor: n}+X_{\left\lceil\frac{n+1}{2}\right\rceil: n}\right)
$$

where $\lfloor x\rfloor$ and $\lceil x\rceil$ denote the floor and the ceiling functions defined as

$$
\lfloor x\rfloor=\max \{n \in \mathbb{Z}: n \leq x\}, \quad\lceil x\rceil=\min \{n \in \mathbb{Z}: x \leq n\} .
$$

The lower (upper) hinge is defined as the median of the lower (upper) half of the sample including sample median. Formally, for simplicity we define

$$
H_{1}=X_{\left\lfloor\frac{n}{4}\right\rfloor+1: n}, \quad H_{2}=X_{n-\left\lfloor\frac{n}{4}\right\rfloor: n} .
$$

Therefore, the trimean of the sample is

$$
T_{n}=\frac{1}{4}\left(X_{\left\lfloor\frac{n}{4}\right\rfloor+1: n}+X_{\left\lfloor\frac{n+1}{2}\right\rfloor: n}+X_{\left\lceil\frac{n+1}{2}\right\rceil: n}+X_{n-\left\lfloor\frac{n}{4}\right\rfloor: n}\right) .
$$


This is an example of "insufficient" but (computationally) quick estimator of $\mu$ and its advantage over the sample median or any other quasimidranges lies in the fact that trimean combines the central tendency of the sample median with the extremes involved in quartiles. For comparison of the trimean with various trimmed means (including sample median) we refer to the paper of Rosenberger and Gasko (1983). Also see Mosteller (2006) for situations when the usage of quick estimators is more appropriate from economical point of view.

Another, heuristical motivation for considering trimean as an estimator of $\mu$ comes from the numerical results presented in the above mentioned papers. If we want to estimate $\mu$ by an $L$-statistic involving as small observations as possible then it appears that, heuristically speaking, the best approximation of $\mu$ by single order statistic is obtained for the sample median, and by two order statistics - for the quasimidrange $M_{r, s}$ with $r \approx \frac{n}{4}$ and $s \approx \frac{3 n}{4}$. Therefore if we want to approximate $\mu$ by linear combination of three order statistics it seems reasonable to use the sample trimean.

In this paper we derive sharp upper and lower bounds for the bias of approximation of $\mu$ by trimean $T_{n}$ expressed in standard deviation units, i.e. on

$$
\frac{E T_{n}-\mu}{\sigma}
$$

Since $T_{n}$ is a symmetric $L$-statistic, then the lower bounds are just negative values of corresponding upper bounds (see Goroncy 2009), so we confine ourselves to the latter ones. The bounds we derive are obtained by the projection method, described in detail in the monograph of Rychlik (2001), which in our case amounts to Moriguti's approach of the greatest convex minorants. This approach has been used in many of the above mentioned papers and also e.g. by Okolewski and Kałuszka (2008) to provide sharp bounds on expectations of concomitants of order statistics.

The main obstacle one has to overcome is to project the function which has three local maxima onto the convex cone $\mathcal{C}$ of nondecreasing square integrable functions on $[0,1]$. Namely, if $f_{i: n}$ denotes the density function of the $i$ th order statistic from uniform $U(0,1)$ distribution, then

$$
E T_{n}=\int_{0}^{1} F^{-1}(u) \varphi_{n}(u) \mathrm{d} u
$$

where

$$
\varphi_{n}(u)=\frac{1}{4}\left(f_{\left\lfloor\frac{n}{4}\right\rfloor+1: n}(u)+f_{\left\lfloor\frac{n+1}{2}\right\rfloor: n}(u)+f_{\left\lceil\frac{n+1}{2}\right\rceil: n}(u)+f_{n-\left\lfloor\frac{n}{4}\right\rfloor: n}(u)\right) .
$$

Therefore, by projection method and Schwartz' inequality

$$
\frac{E T_{n}-\mu}{\sigma} \leq\left\|\bar{\varphi}_{n}-1\right\|_{2}
$$

where $\bar{\varphi}_{n}$ is the projection of $\varphi_{n}$ onto $\mathcal{C}$ (see Rychlik (1998) Thm. 7). The equality is attained for $F$ such that 


$$
\frac{F^{-1}(u)-\mu}{\sigma}=\frac{\bar{\varphi}_{n}(u)-1}{\left\|\bar{\varphi}_{n}-1\right\|_{2}}
$$

It is well-known Moriguti (1953) that the projection $\bar{\varphi}_{n}$ is determined as the righthand derivative of the greatest convex minorant $\bar{\Phi}_{n}$ of the distribution function $\Phi_{n}$ defined as

$$
\Phi_{n}(x)=\int_{0}^{x} \varphi_{n}(u) \mathrm{d} u, \quad x \in[0,1]
$$

Therefore first we are forced in Sect. 2 to determine monotonicity regions of $\varphi_{n}$. However, this is not sufficient in order to find $\bar{\varphi}_{n}$ and in Sect. 3 we consider two auxiliary functions $g_{n}$ and $h_{n}$, which determine the projection $\bar{\varphi}_{n}$ uniquely. In Sect. 4 we apply results of Sect. 3 to determine exact shapes of projections of $\varphi_{n}$ onto $\mathcal{C}$. In Sect. 5 we provide analytical values of bounds on the bias of trimeans, and we illustrate them with their numerical values.

\section{Shapes of projected functions}

In terms of Bernstein polynomials

$$
B_{k, n}(x)=\left(\begin{array}{l}
n \\
k
\end{array}\right) x^{k}(1-x)^{n-k}, \quad x \in[0,1], \quad k=0,1, \ldots, n,
$$

we have $f_{i: n}(u)=n B_{i-1, n-1}(u)$, and putting $j=\left\lfloor\frac{n}{4}\right\rfloor$, we get

$\varphi_{n}(u)= \begin{cases}\frac{n}{4}\left(B_{j, n-1}(u)+2 B_{k, n-1}(u)+B_{n-j-1, n-1}(u)\right), & \text { if } n=2 k+1, \\ \frac{n}{4}\left(B_{j, n-1}(u)+B_{k-1, n-1}(u)+B_{k, n-1}(u)+B_{n-j-1, n-1}(u)\right), & \text { if } n=2 k .\end{cases}$

Using the relation for the derivative of a Bernstein polynomial

$$
B_{k, n}^{\prime}(u)=n\left(B_{k-1, n-1}(u)-B_{k, n-1}(u)\right),
$$

we get for $n=2 k+1$

$$
\begin{aligned}
\varphi_{n}^{\prime}(u)= & \frac{n(n-1)}{4}\left(B_{j-1, n-2}(u)-B_{j, n-2}(u)+2 B_{k-1, n-2}(u)\right. \\
& \left.-2 B_{k, n-2}(u)+B_{n-j-2, n-2}(u)-B_{n-j-1, n-2}(u)\right),
\end{aligned}
$$

and for $n=2 k$

$$
\begin{aligned}
\varphi_{n}^{\prime}(u)= & \frac{n(n-1)}{4}\left(B_{j-1, n-2}(u)-B_{j, n-2}(u)+B_{k-2, n-2}(u)\right. \\
& \left.-B_{k, n-2}(u)+B_{n-j-2, n-2}(u)-B_{n-j-1, n-2}(u)\right) .
\end{aligned}
$$


The sign changes of such linear combinations are studied with the aid of variation diminishing property of Bernstein polynomials of Schoenberg (1959).

Lemma 1 (VDP) The number of zeros in $(0,1)$ of any linear combination $\sum_{i=0}^{n} a_{i} B_{i, n}$ of Bernstein polynomials does not exceed the number of sign changes in the sequence $a_{0}, a_{1}, \ldots, a_{n}$ of its coefficients. Moreover, the first and the last signs of the combination are the same as the signs of the first and the last, respectively, nonzero element of the sequence.

By VDP each $\varphi_{n}^{\prime}$ is either positive-negative $(+-$ for short) or +-+- or +-++- . First we show that the second case is impossible. This follows from part (b) of the next lemma.

Lemma 2 For $n \geq 3$ we have

(a) $\varphi_{n}\left(\frac{1}{2}\right)>1$,

(b) $\varphi_{n}^{\prime \prime}\left(\frac{1}{2}\right)<0$.

The proof of the lemma is given in the Appendix A. Now the function $\varphi_{n}$ is symmetric with respect to $\frac{1}{2}$, so $\varphi_{n}^{\prime}\left(\frac{1}{2}\right)=0$. But $\varphi_{n}$ has maximum at $\frac{1}{2}$ by Lemma 2(b), so it cannot be increasing-decreasing-increasing-decreasing. Therefore, we conclude that $\varphi_{n}^{\prime}$ has either one zero at $\frac{1}{2}$ or it has five zeros $\theta_{1}, \ldots, \theta_{5}$ such that $\theta_{1}<\theta_{2}<\theta_{3}=\frac{1}{2}$ and $\theta_{4}=1-\theta_{2}, \theta_{5}=1-\theta_{5}$.

The shapes of $\varphi_{n}, n \geq 3$, are given in the next Lemma.

Lemma 3 (a) We have $\varphi_{n}(0)=\varphi_{n}(1)=0$.

(b) For $3 \leq n \leq 8$, the function $\varphi_{n}$ is increasing-decreasing.

(c) For $n \geq 9$, the function $\varphi_{n}$ is either increasing-decreasing with maximum at $\frac{1}{2}$ or it has three local maxima (one of them at $x=\frac{1}{2}$ and two remaining at points symmetric with respect to $1 / 2$ ) and two local minima.

Now, if $\varphi_{n}$ is increasing-decreasing, then it is well known that the projection $\bar{\varphi}_{n}$ is of the form

$$
\bar{\varphi}_{n}(u)= \begin{cases}\varphi_{n}(u), & \text { for } \quad 0 \leq u \leq \alpha \\ \varphi_{n}(\alpha), & \text { for } \quad \alpha \leq u \leq 1\end{cases}
$$

where $\alpha$ is the only solution to the equation

$$
1-\Phi_{n}(\alpha)=(1-\alpha) \varphi_{n}(\alpha), \quad \alpha \in(0,1) \text {. }
$$

However, if $\varphi_{n}^{\prime}$ has five zeros, then the derivation of $\bar{\varphi}_{n}$ is much more complicated, and it will be done in the next section with the aid of some auxiliary functions.

\section{Auxiliary functions}

To determine $\bar{\varphi}_{n}$ we need to find the greatest convex minorant $\bar{\Phi}_{n}$ of $\Phi_{n}$. It is a little bit easier to determine the greatest convex minorant $\bar{\Psi}_{n}$ of the function $\Psi_{n}$ defined as

$$
\Psi_{n}(u)=\Phi_{n}(u)-u, \quad 0 \leq u \leq 1 .
$$


Then $\Psi_{n}(0)=\Psi_{n}(1)=0$, and $\bar{\varphi}_{n}=\bar{\Psi}_{n}^{\prime}+1$.

Next, we define and analyze two auxiliary functions $g_{n}$ and $h_{n}$ introduced by Bieniek (2014b) for quasimidranges. Let $\ell_{\alpha}$ denote the straight line which is tangent to $\Psi_{n}$ at the point $\alpha \in[0,1]$, i.e.

$$
\ell_{\alpha}(x)=\Psi_{n}(\alpha)+\left(\varphi_{n}(\alpha)-1\right)(x-\alpha), \quad x \in \mathbb{R} .
$$

Let $g_{n}(\alpha)$ denote the value of $\ell_{\alpha}$ at $x=1$, i.e.

$$
g_{n}(\alpha)=\Phi_{n}(\alpha)+(1-\alpha) \varphi_{n}(\alpha)-1
$$

and $h_{n}(\alpha)$ denote the slope of the straight line passing through the points $\left(\alpha, \Phi_{n}(\alpha)\right)$ and $(1,1)$, i.e.

$$
h_{n}(\alpha)=\frac{1-\Phi_{n}(\alpha)}{1-\alpha} .
$$

Let us study the properties of the function $g_{n}$. We have $g_{n}(0)=\varphi_{n}(0)-1=-1$ and $g_{n}(1)=0$. Next, easy differentiation leads to

$$
g_{n}(u)=(1-u) \varphi_{n}^{\prime}(u)
$$

and so $g_{n}$ has the same monotonicity properties as $\varphi_{n}$. Now we find the number of zeros of $g_{n}$ in $(0,1)$. Note that $g_{n}(\alpha)=0$ if and only if $\alpha$ satisfies (5).

Theorem 1 The function $g_{n}$ has either one or three or five zeros in $(0,1)$.

Proof We consider the case of odd $n=2 k+1, k \geq 3$. The case of even $n$ can be treated analogously. Let $j=\left\lfloor\frac{n}{4}\right\rfloor$. We start with the representation of the distribution function $F_{k: n}$ of $k$ th order statistic from uniform distribution on $[0,1]$

$$
F_{k: n}(u)=\sum_{i=k}^{n} B_{i, n}(u), \quad u \in[0,1] .
$$

Therefore

$$
\begin{aligned}
\Phi_{n}(u) & =\frac{1}{4}\left(F_{j+1: n}(u)+2 F_{k+1: n}(u)+F_{n-j: n}(u)\right) \\
& =\frac{1}{4} \sum_{i=j+1}^{k} B_{i, n}(u)+\frac{3}{4} \sum_{i=k+1}^{n-j-1} B_{i, n}(u)+\sum_{i=n-j}^{n} B_{i, n}(u) .
\end{aligned}
$$

Next, using the relation

$$
(1-t) B_{i, n-1}(t)=\frac{n-i}{n} B_{i, n}(t)
$$


we derive

$$
(1-u) \varphi_{n}(u)=\frac{1}{4}\left[(n-j) B_{j, n}(u)+2(n-k) B_{k, n}(u)+(j+1) B_{n-j-1, n}(u)\right] .
$$

Finally by binomial theorem we have $\sum_{i=0}^{n} B_{i, n}(u)=1$ for $0 \leq u \leq 1$, and combining this with Eqs. (6) and (8), after some algebra we get

$$
\begin{aligned}
g_{n}(u)= & -\sum_{i=0}^{j-1} B_{i, n}(u)+\frac{n-j-4}{4} B_{j, n}(u)-\frac{3}{4} \sum_{i=j+1}^{k-1} B_{i, n}(u) \\
& +\frac{2 n-2 k-3}{4} B_{k, n}(u)-\frac{1}{4} \sum_{i=k+1}^{n-j-2} B_{i, n}(u)+\frac{j}{4} B_{n-j-1, n}(u) .
\end{aligned}
$$

The conclusion of the theorem follows by VDP.

Finally we find the locations of zeros of $g_{n}$ in $[0,1]$. Note that if $\varphi_{n}^{\prime}$ has five zeros $\theta_{1}, \ldots, \theta_{5}$ then necessarily $g_{n}\left(\theta_{5}\right)>0$, so due to its monotonicity properties $g_{n}$ may have at most one zero in each of the intervals $\left(0, \theta_{1}\right),\left(\theta_{1}, \theta_{2}\right), \ldots\left(\theta_{4}, \theta_{5}\right)$ and no zeros in $\left(\theta_{5}, 1\right)$.

Lemma 4 (a) For all $n \geq 3$ we have $g_{n}\left(\frac{1}{2}\right)>0$;

(b) If $\varphi_{n}^{\prime}$ has five zeros, then $g_{n}\left(\theta_{4}\right)>g_{n}\left(\theta_{2}\right)$.

(c) If $g_{n}$ has a root $\alpha_{2} \in\left(\theta_{1}, \theta_{2}\right)$, then it must have roots $\alpha_{1} \in\left(0, \theta_{1}\right)$ and $\alpha_{3} \in\left(\theta_{2}, \theta_{3}\right)$.

(d) If $g_{n}$ has a root in $\left(\theta_{3}, \theta_{5}\right)$, then it has exactly two roots in this interval, and one root in $\left(\theta_{2}, \theta_{3}\right)$.

Proof (a) We have $g_{n}\left(\frac{1}{2}\right)=\frac{1}{2}\left(\varphi_{n}\left(\frac{1}{2}\right)-1\right)>0$ by Lemma 2(a).

(b) Since $\varphi_{n}$ is symmetric with respect to $\frac{1}{2}$ then $\varphi_{n}\left(\theta_{2}\right)=\varphi_{n}\left(\theta_{4}\right)$ and $\varphi_{n}(u)>\varphi_{n}\left(\theta_{2}\right)$ for $u \in\left(\theta_{2}, \theta_{4}\right)$. Therefore

$$
\Phi_{n}\left(\theta_{4}\right)-\Phi_{n}\left(\theta_{2}\right)=\int_{\theta_{2}}^{\theta_{4}} \varphi_{n}(u) \mathrm{d} u>\varphi_{n}\left(\theta_{2}\right)\left(\theta_{4}-\theta_{2}\right)
$$

and

$$
\begin{aligned}
g_{n}\left(\theta_{4}\right)= & \Phi_{n}\left(\theta_{2}\right)+\left(\Phi_{n}\left(\theta_{4}\right)-\Phi_{n}\left(\theta_{2}\right)\right)+\left(1-\theta_{4}\right) \varphi_{n}\left(\theta_{4}\right)-1 \\
& >\Phi_{n}\left(\theta_{2}\right)+\varphi_{n}\left(\theta_{2}\right)\left(\theta_{4}-\theta_{2}\right)+\left(1-\theta_{4}\right) \varphi_{n}\left(\theta_{2}\right)-1=g_{n}\left(\theta_{2}\right) .
\end{aligned}
$$

(c) If $g_{n}\left(\alpha_{2}\right)=0$ for some $\alpha_{2} \in\left(\theta_{1}, \theta_{2}\right)$, then $g_{n}\left(\theta_{1}\right)>0$ and $g_{n}\left(\theta_{2}\right)<0$. The conclusion follows from $g_{n}(0)<0$ and $g_{n}\left(\theta_{3}\right)>0$ (see part (a) of this lemma).

(d) Since $g_{n}\left(\theta_{3}\right)>0$ and $g_{n}\left(\theta_{5}\right)>0$, the function $g_{n}$ has even number of zeros in $\left(\theta_{3}, \theta_{5}\right)$, so it has at least two zeros. Since $g_{n}$ is decreasing-increasing on $\left(\theta_{3}, \theta_{5}\right)$, it may have at most two zeros in the interval.

If $g_{n}$ has a root in $\left(\theta_{3}, \theta_{5}\right)$, then $g_{n}\left(\theta_{4}\right)<0$, and so by part (b) of the lemma we have $g\left(\theta_{2}\right)<0$, and $g_{n}$ has another root in $\left(\theta_{2}, \theta_{3}\right)$. 
For simplicity denote $\theta_{0}=0$. By Lemma 4 the locations of zeros of $g_{n}$ are as follows.

Corollary 1 (a) If $g_{n}$ has exactly one zero $\alpha_{1}$, then $\alpha_{1} \in\left(0, \theta_{1}\right) \cup\left(\theta_{2}, \theta_{3}\right)$.

(b) If $g_{n}$ has exactly three zeros $\alpha_{1}, \alpha_{2}, \alpha_{3}$, then either $\alpha_{i} \in\left(\theta_{i-1}, \theta_{i}\right)$ for $i=1,2,3$, or $\alpha_{1} \in\left(\theta_{2}, \theta_{3}\right), \alpha_{2} \in\left(\theta_{3}, \theta_{4}\right)$ and $\alpha_{3} \in\left(\theta_{4}, \theta_{5}\right)$.

(c) If $g_{n}$ has five zeros $\alpha_{1}, \ldots, \alpha_{5}$, then $\alpha_{i} \in\left(\theta_{i-1}, \theta_{i}\right)$ for $i=1, \ldots, 5$.

Now we study some properties of $h_{n}$. First of all, $h_{n}(0)=1$ and $h(1)=0$. Moreover,

$$
h_{n}^{\prime}(u)=-\frac{g_{n}(u)}{(1-u)^{2}},
$$

so the monotonicity properties of $h_{n}$ are determined by the signs of $g_{n}$. Before we determine the shapes of $h_{n}$ first we study the number of solutions to $h_{n}(u)=1$ in $(0,1)$. Clearly we have $h_{n}\left(\frac{1}{2}\right)=1$ and in the next lemma we prove that this is the only solution.

Lemma 5 The equation $h_{n}(u)=1$ has unique root $u=\frac{1}{2}$ in $(0,1)$. Moreover $h_{n}(u)>1$ if and only if $u \in\left(0, \frac{1}{2}\right)$.

Proof We again consider only the case of odd $n=2 k+1$, and the case of $n$ even is left for the reader. Using (6) and (7) after some algebra we obtain

$$
h_{n}(u)=\sum_{i=0}^{j} \frac{n}{n-i} B_{i, n-1}(u)+\frac{3}{4} \sum_{i=j+1}^{k} B_{i, n-1}(u)+\frac{1}{4} \sum_{i=k+1}^{n-j-1} \frac{n}{n-i} B_{i, n-1}(u) .
$$

This time we write $1=\sum_{i=0}^{n-1} B_{i, n-1}(u)$ which combined with the last equality yields

$$
h_{n}(u)-1=\sum_{i=0}^{n-1} a_{i} B_{i, n-1}(u)
$$

where

$$
a_{i}= \begin{cases}\frac{i}{n-i}, & \text { for } 0 \leq i \leq j \\ \frac{4 i-n}{4(n-i)}, & \text { for } j+1 \leq i \leq k \\ \frac{4 i-3 n}{4(n-i)}, & \text { for } k+1 \leq i \leq n-j-1 \\ -1, & \text { for } n-j \leq i \leq n-1\end{cases}
$$

We have $a_{j+1}>\ldots>a_{k}$ and $a_{n-j-1}<\ldots<a_{k+1}$, and since $k=\lfloor n / 2\rfloor$, we easily prove that $a_{k}>0$ and $a_{k+1}<0$. Therefore $a_{i}>0$ for $0 \leq i \leq k$, and $a_{i}<0$ for $k+1 \leq i \leq n-1$. By VDP the function $h_{n}(u)-1$ has exactly one zero in $(0,1)$.

To prove the second statement it suffices to note that $g_{n}$ is first negative, so $h_{n}$ is first increasing by (10). Since $h_{n}(0)=1$, then $h_{n}(u)>1$ in a neighbourhood of 0 . But by part (a) of this lemma, this must be $\left(0, \frac{1}{2}\right)$. Moreover $g_{n}$ has at least one zero in 
$\left(0, \frac{1}{2}\right)$ and $g_{n}\left(\frac{1}{2}\right)>0$, so $g_{n}$ is positive in a neighbourhood of $\frac{1}{2}$, and $h_{n}$ is decreasing there. This implies that $h_{n}(u)<1$ for $u \in\left(\frac{1}{2}, 1\right)$.

Corollary 2 The function $\Psi_{n}$ has exactly one root at $\frac{1}{2}$ in $(0,1)$, and it is negative in $\left(0, \frac{1}{2}\right)$, and positive in $\left(\frac{1}{2}, 1\right)$.

Proof It suffices to note that $h_{n}(u)>1$ if and only if $\Psi_{n}(u)>0$, so the signs of $h_{n}-1$ and $\Psi_{n}$ are the same.

Now we can determine extrema of $h_{n}$.

Theorem 2 (a) If $g_{n}$ has exactly one zero $\alpha_{1}$, then $h_{n}$ has global maximum at $\alpha_{1}$.

(b) If $g_{n}$ has exactly three zeros $\alpha_{1}, \alpha_{2}, \alpha_{3}$, then $h_{n}$ has local maxima at $\alpha_{1}$ and $\alpha_{3}$ with global maximum inside $\left(0, \frac{1}{2}\right)$.

(c) If $g_{n}$ has five zeros $\alpha_{1}, \ldots, \alpha_{5}$, then $h_{n}$ has local maxima at $\alpha_{1}, \alpha_{3}, \alpha_{5}$ with global maximum at $\alpha_{1}$ or $\alpha_{3}$.

Proof If $g_{n}$ has exactly one zero, then $g_{n}$ is negative on $\left(0, \alpha_{1}\right)$, and positive otherwise, so by (10), the function $h_{n}$ is increasing on $\left(0, \alpha_{1}\right)$ and decreasing on $\left(\alpha_{1}, 1\right)$. This proves part (a).

Similar analysis proves (b) and (c) except for the location of global maximum. But it suffices to note that by Lemma 5 we have $h_{n}(u)>1>h_{n}(v)$ for $u \in\left(0, \frac{1}{2}\right)$ and $v \in\left(\frac{1}{2}, 1\right)$.

We close this section with some properties of $g_{n}$ and $h_{n}$ useful in the next section.

Lemma 6 (a) If $g_{n}(\alpha)=0$, then $h_{n}(\alpha)=\varphi_{n}(\alpha)$.

(b) If $g_{n}(\alpha)=0$ and $h_{n}(\alpha) \geq h_{n}(u)$ for $u \in(\alpha, 1)$, then $\Psi_{n}(u) \geq \ell_{\alpha}(u)$ for $u \in(\alpha, 1)$.

(c) If $\theta$ is any root of $\varphi_{n}^{\prime}$ with $g_{n}(\theta)<0$, and $\alpha$ is the smallest zero of $g_{n}$ in $(\theta, 1)$, then $\varphi_{n}(\theta)<\varphi_{n}(\alpha)$.

Proof Part (a) is just the definition of $h_{n}$ and $g_{n}$.

To prove part (b) recall that if $g_{n}(\alpha)=0$, then the tangent to $\Psi_{n}$ at $\alpha$ passes through $(1,0)$, so $\ell_{\alpha}$ can be written as

$$
\ell_{\alpha}(u)=\left(h_{n}(\alpha)-1\right)(u-1) .
$$

Since $h_{n}(\alpha) \geq h_{n}(u)$ for $u \in[\alpha, 1]$, then

$$
\ell_{\alpha}(u) \leq\left(h_{n}(u)-1\right)(u-1)=\Psi_{n}(u)
$$

To prove (c) it suffices to note that

$$
\varphi_{n}(\theta)<h_{n}(\theta)<h_{n}(\alpha)=\varphi_{n}(\alpha)
$$

The first inequality follows from $g_{n}(\theta)<0$, the second follows from the fact that $h_{n}$ is increasing on $(\theta, \alpha)$, and the last equality follows from part (a) of the lemma. 


\section{Shapes of projections}

Now we can determine the projections of $\varphi_{n}, n \geq 3$. In the proof of the main result of this section we need the following lemma. It was implicitly stated and proved in the proof of Theorem 4.1 of Bieniek (2014b), but here for the convenience of the reader we state and prove it in a little bit more abstract setting.

Consider any continuous function $\phi:\left(0, \frac{1}{2}\right) \rightarrow[0, \infty)$ with the following properties: $\phi(0)=0$, and there exist $\theta_{1}, \theta_{2} \in\left(0, \frac{1}{2}\right)$ such that $\phi$ is strictly increasing on $\left(0, \theta_{1}\right)$ and $\left(\theta_{2}, \frac{1}{2}\right)$, and strictly decreasing on $\left(\theta_{1}, \theta_{2}\right)$ with $\phi\left(\theta_{1}\right)<\phi\left(\frac{1}{2}\right)$ and $\phi\left(\theta_{2}\right)>0$. Let

$$
\Phi(x)=\int_{0}^{x} \phi(t) \mathrm{d} t, \quad 0 \leq x \leq \frac{1}{2}
$$

denote the antiderivative of $\phi$.

Let $\beta_{0}$ denote the unique point of $\left(0, \theta_{1}\right)$ such that $\phi\left(\beta_{0}\right)=\phi\left(\theta_{2}\right)$, and fix any $\eta \in\left(\beta_{0}, \theta_{1}\right]$. Let $\gamma_{0}$ be the unique point of $\left(\theta_{2}, \frac{1}{2}\right)$ such that $\phi\left(\gamma_{0}\right)=\phi(\eta)$. Then for every $\beta \in\left[\beta_{0}, \eta\right]$ there exists the unique $\gamma=\gamma(\beta) \in\left[\theta_{2}, \gamma_{0}\right]$ such that $\phi(\gamma)=\phi(\beta)$. Therefore the following function

$$
k(\beta)=\phi(\beta)(\gamma-\beta)-(\Phi(\gamma)-\Phi(\beta)), \quad \beta \in\left[\beta_{0}, \alpha\right],
$$

is well-defined.

Lemma 7 If $k(\eta)>0$, then the function $k$ has exactly one zero in $\left(\beta_{0}, \eta\right)$.

Note that $k(\beta)=0$ is equivalent to the system of equations

$$
\frac{\Phi(\gamma)-\Phi(\beta)}{\gamma-\beta}=\phi(\beta)=\phi(\gamma) .
$$

Proof Since the function $k$ is continuous, it suffices to prove that $k$ is strictly increasing with $k\left(\beta_{0}\right)<0$.

Firstly, since $\phi(u)>\phi\left(\beta_{0}\right)$ for $u \in\left(\beta_{0}, \theta_{2}\right)$, then

$$
\begin{aligned}
\Phi\left(\theta_{2}\right) & =\int_{0}^{\theta_{2}} \phi(u) \mathrm{d} u>\int_{0}^{\beta_{0}} \phi(u) \mathrm{d} u+\left(\theta_{2}-\beta_{0}\right) \phi\left(\beta_{0}\right) \\
& =\Phi\left(\beta_{0}\right)+\left(\theta_{2}-\beta_{0}\right) \phi\left(\beta_{0}\right)
\end{aligned}
$$

and therefore $k\left(\beta_{0}\right)<0$.

Secondly, for given $\beta_{1}<\beta_{2}$ and $\gamma_{1}<\gamma_{2}$, where $\phi\left(\beta_{i}\right)=\phi\left(\gamma_{i}\right), i=1,2$, there exist unique $\delta_{1}, \delta_{2} \in\left(\theta_{1}, \theta_{2}\right)$ such that $\phi\left(\delta_{i}\right)=\phi\left(\beta_{i}\right), i=1,2$. Clearly $\delta_{2}<\delta_{1}$. On each of the intervals $\left(\beta_{1}, \beta_{2}\right)$ and $\left(\delta_{2}, \theta_{1}\right)$ we have $\phi(u)>\phi\left(\beta_{1}\right)$, so 


$$
\begin{aligned}
\left(\Phi\left(\delta_{1}\right)-\Phi\left(\beta_{1}\right)\right)-\left(\Phi\left(\delta_{2}\right)-\Phi\left(\beta_{2}\right)\right)= & \int_{\beta_{1}}^{\beta_{2}} \phi(u) \mathrm{d} u+\int_{\delta_{2}}^{\delta_{1}} \phi(u) \mathrm{d} u \\
& >\phi\left(\beta_{1}\right)\left[\left(\beta_{2}-\beta_{1}\right)+\left(\delta_{1}-\delta_{2}\right)\right] \\
& >\phi\left(\beta_{1}\right)\left(\delta_{1}-\beta_{1}\right)-\phi\left(\beta_{2}\right)\left(\delta_{2}-\beta_{2}\right)
\end{aligned}
$$

since $\phi\left(\beta_{2}\right)>\phi\left(\beta_{1}\right)$. Therefore

$$
\phi\left(\beta_{1}\right)\left(\delta_{1}-\beta_{1}\right)-\left(\Phi\left(\delta_{1}\right)-\Phi\left(\beta_{1}\right)\right)<\phi\left(\beta_{2}\right)\left(\delta_{2}-\beta_{2}\right)-\left(\Phi\left(\delta_{2}\right)-\Phi\left(\beta_{2}\right)\right) .
$$

Similarly,

$$
\phi\left(\beta_{1}\right)\left(\gamma_{1}-\delta_{1}\right)-\left(\Phi\left(\gamma_{1}\right)-\Phi\left(\delta_{1}\right)\right)<\phi\left(\beta_{2}\right)\left(\gamma_{2}-\delta_{2}\right)-\left(\Phi\left(\gamma_{2}\right)-\Phi\left(\delta_{2}\right)\right) .
$$

Summing up both inequalities side by side we conclude that $k\left(\beta_{1}\right)<k\left(\beta_{2}\right)$, so $k$ is strictly increasing.

The main result of this section is the following theorem.

Theorem 3 (a) If either of the following conditions hold

- $g_{n}$ has exactly one root $\alpha_{1} \in\left(0, \theta_{1}\right)$,

- $g_{n}$ has at least three roots $\alpha_{1}, \alpha_{2}, \alpha_{3} \in\left(0, \frac{1}{2}\right)$ with $h_{n}\left(\alpha_{1}\right) \geq h_{n}\left(\alpha_{3}\right)$, then

$$
\bar{\varphi}_{n}(u)= \begin{cases}\varphi_{n}(u), & \text { for } 0 \leq u \leq \alpha_{1}, \\ \varphi_{n}\left(\alpha_{1}\right), & \text { for } \alpha_{1} \leq u \leq 1\end{cases}
$$

(b) Otherwise, i.e. if one of the following conditions hold

- $g_{n}$ has exactly one root $\alpha_{3} \in\left(\theta_{2}, \theta_{3}\right)$,

- $g_{n}$ has exactly three roots $\alpha_{3}, \alpha_{4}, \alpha_{5} \in\left(\theta_{2}, 1\right)$,

- $g_{n}$ has at least three roots $\alpha_{1}, \alpha_{2}, \alpha_{3} \in\left(0, \frac{1}{2}\right)$ with $h_{n}\left(\alpha_{1}\right)<h_{n}\left(\alpha_{3}\right)$, then

$$
\bar{\varphi}_{n}(u)= \begin{cases}\varphi_{n}(u), & \text { for } 0 \leq u \leq \beta, \\ \varphi_{n}(\beta), & \text { for } \beta \leq u \leq \gamma, \\ \varphi_{n}(u), & \text { for } \gamma \leq u \leq \alpha_{3}, \\ \varphi_{n}\left(\alpha_{3}\right), & \text { for } \alpha_{3} \leq u \leq 1,\end{cases}
$$

where $(\beta, \gamma)$, with $\beta \in\left(0, \theta_{1}\right)$ and $\gamma \in\left(\theta_{2}, \alpha_{3}\right)$, is the unique solution to the system of equations

$$
\frac{\Phi_{n}(\gamma)-\Phi_{n}(\beta)}{\gamma-\beta}=\varphi_{n}(\beta)=\varphi_{n}(\gamma) .
$$

Proof First we consider the case when $g_{n}$ has a single root in $(0,1)$. 
If $g_{n}(\alpha)=0$ for unique $\alpha \in\left(0, \theta_{1}\right)$, then $\alpha$ is a point of global maximum of $h_{n}$. Then $\Psi_{n}$ is convex on $(0, \alpha)$, and by Lemma $6(\mathrm{~b})$ we have $\Psi_{n}(u) \geq \ell_{\alpha}(u)$ for $u \in[\alpha, 1]$. Therefore

$$
\bar{\Psi}_{n}(u)= \begin{cases}\Psi_{n}(u), & \text { for } 0 \leq u \leq \alpha \\ \ell_{\alpha}(u), & \text { for } \alpha \leq u \leq 1\end{cases}
$$

is the greatest convex minorant of $\Psi_{n}$, and $\bar{\varphi}_{n}$ is of the form (13).

If $g_{n}(\alpha)=0$ for unique $\alpha \in\left(\theta_{2}, \theta_{3}\right)$, then also $\Psi_{n}(u) \geq \ell_{\alpha}(u)$ for $u \geq \alpha$, but $\Psi_{n}$ is not convex on $(0, \alpha)$. But then $g_{n}\left(\theta_{1}\right)<0$, so by Lemma 6(c) we have $\varphi_{n}\left(\theta_{1}\right) \leq$ $\varphi_{n}(\alpha)$. Now apply Lemma 7 with $\eta=\theta_{1}$ and $\phi=\varphi_{n}$. We have $\varphi_{n}(u)<\varphi_{n}\left(\theta_{1}\right)$ for $u \in\left(\theta_{1}, \gamma_{1}\right)$, so similar computations as in (12) imply that $k\left(\theta_{1}\right)>0$. Therefore there exists the unique pair $(\beta, \gamma)$ which satisfies the system (15). Now the function

$$
\bar{\Psi}_{n}(u)= \begin{cases}\Psi_{n}(u), & \text { for } 0 \leq u \leq \beta \text { or } \gamma \leq u \leq \alpha, \\ \ell_{\beta}(u), & \text { for } \beta \leq u \leq \gamma \\ \ell_{\alpha}(u), & \text { for } \alpha \leq u \leq 1,\end{cases}
$$

is the greatest convex minorant of $\Psi_{n}$, and $\bar{\varphi}_{n}$ is given by (14).

Next, we consider the case of three zeros of $g_{n}$ with exactly one $\alpha \in\left(0, \frac{1}{2}\right)$ and two of them $\alpha_{4}, \alpha_{5} \in\left(\frac{1}{2}, 1\right)$. Then $h_{n}$ has two local maxima at $\alpha$ and $\alpha_{5}$ with $\alpha$ being global maximum. By Corollary 1 (b) we have $\alpha \in\left(\theta_{2}, \theta_{3}\right)$, so again $g_{n}\left(\theta_{1}\right)<0$, and it suffices to repeat the reasoning for the case of exactly one zero of $g_{n}$ belonging to $\left(\theta_{2}, \theta_{3}\right)$.

Finally, we turn to the case of three zeros of $g_{n}$ inside $\left(0, \frac{1}{2}\right)$ (and possibly two zeros in $\left.\left(\frac{1}{2}, 1\right)\right)$. If $h_{n}\left(\alpha_{1}\right) \geq h_{n}\left(\alpha_{3}\right)$, then $\alpha_{1}$ is the point of global maximum of $g_{n}$, and again the greatest convex minorant of $\Psi_{n}$ is given by (16), and the projection of $\varphi_{n}$ is of the form (13).

However, if $h_{n}\left(\alpha_{1}\right)<h_{n}\left(\alpha_{3}\right)$, then $\alpha_{3}$ is the point of global minimum of $h_{n}$ and more thorough analysis is needed. By Theorem 2(b) and (c) the function $h_{n}$ has local minimum at $\alpha_{2}$, so $h_{n}\left(\alpha_{1}\right)>h_{n}\left(\alpha_{2}\right)$. By Lemma 6(a) the last two conditions are equivalent to $\varphi_{n}\left(\alpha_{2}\right)<\varphi_{n}\left(\alpha_{1}\right)<\varphi_{n}\left(\alpha_{3}\right)$. Moreover, obviously $\varphi_{n}\left(\alpha_{2}\right)>\varphi_{n}\left(\theta_{2}\right)$, so $\varphi_{n}\left(\alpha_{1}\right)>\varphi_{n}\left(\theta_{2}\right)$ as well. Now apply Lemma 7 with $\eta=\alpha_{1}$ and $\phi=\varphi_{n}$. Recall that $\alpha_{1}$ satisfies the equation (5), so

$$
\left(1-\gamma_{0}\right) \varphi_{n}\left(\alpha_{1}\right)=1-\Phi_{n}\left(\alpha_{1}\right)-\left(\gamma_{0}-\alpha_{1}\right) \varphi_{n}\left(\alpha_{1}\right)
$$

and therefore $g_{n}\left(\gamma_{0}\right)=-k\left(\alpha_{1}\right)$. But $\gamma_{0} \in\left(\alpha_{2}, \alpha_{3}\right)$, so $g_{n}\left(\gamma_{0}\right)<0$, and $k\left(\alpha_{1}\right)>0$, and application of Lemma 7 completes the proof of the theorem. 


\section{Analytical and numerical values of bounds}

Once the projections of $\varphi_{n}$ onto $\mathcal{C}$ are found, the determination of values of the bounds

$$
B_{n}=\sup _{F} \frac{E T_{n}-\mu}{\sigma}
$$

as well as the conditions for their attainability, is easy due to (1) and (2). Therefore the proof of the next result is omitted.

Theorem 4 If any of the conditions of Theorem 3(a) holds, then

$$
B_{n}=\left(\int_{0}^{\alpha}\left(\varphi_{n}(u)\right)^{2} \mathrm{~d} u+(1-\alpha)\left(\varphi_{n}(\alpha)\right)^{2}-1\right)^{1 / 2},
$$

where $\alpha$ is the unique zero of $g_{n}$ in $\left(0, \theta_{1}\right)$. Otherwise,

$$
\begin{aligned}
B_{n}= & \left(\int_{0}^{\beta}\left(\varphi_{n}(u)\right)^{2} \mathrm{~d} u+(\gamma-\beta)\left(\varphi_{n}(\beta)\right)^{2}\right. \\
& \left.+\int_{\beta}^{\alpha}\left(\varphi_{n}(u)\right)^{2} \mathrm{~d} u+(1-\alpha)\left(\varphi_{n}(\alpha)\right)^{2}-1\right)^{1 / 2},
\end{aligned}
$$

where $\alpha$ is the unique zero of $g_{n}$ in $\left(\theta_{2}, \theta_{3}\right)$, and $(\beta, \gamma)$ is the unique solution to (14). In both cases the equality is attained for the distribution function $F$ given by

$$
F(x)= \begin{cases}0, & \text { if } \frac{x-\mu}{\sigma}<-\frac{1}{B_{n}} \\ \varphi_{n}^{-1}\left(1+B_{n} \frac{x-\mu}{\sigma}\right), & \text { if }-\frac{1}{B_{n}} \leq \frac{x-\mu}{\sigma}<\frac{\varphi_{n}(\alpha)-1}{B_{n}} \\ 1, & \text { if } \frac{x-\mu}{\sigma} \geq \frac{\varphi_{n}(\alpha)-1}{B_{n}}\end{cases}
$$

Remark 1 The inverse $\varphi_{n}^{-1}$ of $\varphi_{n}$ should be understood as the inverse of the function $\varphi_{n}$ restricted to the interior of the set where $\bar{\varphi}_{n}=\varphi_{n}$. Note that in the second case the distribution function $F$ attaining the bound has the jump of size $\gamma-\beta$ at $x=\frac{\varphi_{n}(\gamma)-1}{B_{n}}$.

Remark 2 The results of Theorem 4 can be generalized to provide bounds expressed in scale units of $p$ th central absolute moment with arbitrary $p \in[1, \infty]$ instead of $p=2$ only.

We conclude the paper with numerical values of bounds of Theorem 4, which are presented in Table 1. Note that by (9) and (11) the functions $g_{n}$ and $h_{n}$ are polynomials of the degree at most $n$, so numerical verification of the conditions of Theorem 3 is straightforward. Quite surprisingly, $B_{n}, n \geq 3$, is not monotone sequence, but it can be observed that each of the sequences $B_{4 k}, B_{4 k+1}, B_{4 k+2}$ and $B_{4 k+3}, k \geq 1$, is strictly increasing. 
Table 1 The values of the bounds $B_{n}, 3 \leq n \leq 20$

\begin{tabular}{lllllll}
\hline$n$ & 3 & 4 & 5 & 6 & 7 & 8 \\
\hline$B_{n}$ & 0.0677 & 0.2710 & 0.2872 & 0.2481 & 0.2286 & 0.3541 \\
$n$ & 9 & 10 & 11 & 12 & 13 & 14 \\
$B_{n}$ & 0.3374 & 0.3075 & 0.2855 & 0.3789 & 0.3591 & 0.3357 \\
$n$ & 15 & 16 & 17 & 18 & 19 & 20 \\
$B_{n}$ & 0.3161 & 0.3903 & 0.3719 & 0.3529 & 0.3364 & 0.3971 \\
\hline
\end{tabular}

Open Access This article is distributed under the terms of the Creative Commons Attribution License which permits any use, distribution, and reproduction in any medium, provided the original author(s) and the source are credited.

\section{Appendix}

\section{Proof of Lemma 2}

Proof (a) We need to prove that $\varphi_{n}\left(\frac{1}{2}\right)>1$. For $n=3, \ldots, 6$ this follows from the fact that $\varphi_{n}$ is increasing-decreasing with maximum at $\frac{1}{2}$. For $n=2 k+1, k \geq 3$, by Lemma 2.3 of Bieniek (2014b) we have $f_{k+1: 2 k+1}\left(\frac{1}{2}\right)>2$. Therefore

$$
\varphi_{2 k+1}\left(\frac{1}{2}\right) \geq \frac{1}{2} f_{k+1: 2 k+1}\left(\frac{1}{2}\right)>1 .
$$

For $n=2 k, k \geq 4$, by Lemma A.3 of Bieniek (2014b) we have $f_{k: 2 k}\left(\frac{1}{2}\right)=$ $f_{k+1: 2 k}\left(\frac{1}{2}\right)>2$, and therefore

$$
\varphi_{2 k}\left(\frac{1}{2}\right) \geq \frac{1}{4}\left(f_{k: 2 k}\left(\frac{1}{2}\right)+f_{k+1: 2 k}\left(\frac{1}{2}\right)\right)>1 .
$$

Proof (b) For $n=3, \ldots, 8$ the statement follows from the fact that $\frac{1}{2}$ is the point of maximum of $\varphi_{n}$.

We give the detailed proof for the case $n=4 j+1, j \geq 2$, only. The proofs for the remaining cases are analogous. Differentiating (4) with the aid of (3), and putting $u=\frac{1}{2}$ we obtain

$$
\begin{aligned}
\varphi_{4 j+1}^{\prime \prime}\left(\frac{1}{2}\right)= & \frac{4 j(4 j+1)(4 j-1)}{2^{4 j-1}}\left[\left(\begin{array}{c}
4 j-2 \\
j-2
\end{array}\right)-2\left(\begin{array}{c}
4 j-2 \\
j-1
\end{array}\right)\right. \\
& \left.+\left(\begin{array}{c}
4 j-2 \\
j
\end{array}\right)+2\left(\begin{array}{c}
4 j-2 \\
2 j-2
\end{array}\right)-2\left(\begin{array}{c}
4 j-2 \\
2 j-1
\end{array}\right)\right] .
\end{aligned}
$$

Therefore $\varphi_{9}^{\prime \prime}\left(\frac{1}{2}\right)<0$, and it remains to consider $j \geq 3$. By the above equality we get

$$
\varphi_{4 j+1}^{\prime \prime}\left(\frac{1}{2}\right)<0 \Longleftrightarrow A<2 B+C,
$$


where

$$
\begin{aligned}
& A=\left(\begin{array}{c}
4 j-2 \\
j
\end{array}\right)-\left(\begin{array}{c}
4 j-2 \\
j-1
\end{array}\right)=\frac{(4 j-2) !}{j !(3 j-1) !}(2 j-1), \\
& B=\left(\begin{array}{c}
4 j-2 \\
2 j-1
\end{array}\right)-\left(\begin{array}{c}
4 j-2 \\
2 j-2
\end{array}\right)=\frac{(4 j-2) !}{j !(3 j-1) !} \frac{(2 j) \ldots(3 j-1)}{(j+1) \ldots(2 j-1)}, \\
& C=\left(\begin{array}{c}
4 j-2 \\
j-1
\end{array}\right)-\left(\begin{array}{c}
4 j-2 \\
j-2
\end{array}\right)=\frac{(4 j-2) !}{j !(3 j-1) !} \frac{2 j+1}{3} .
\end{aligned}
$$

Therefore $\varphi_{4 j+1}^{\prime \prime}\left(\frac{1}{2}\right)<0$ is equivalent to

$$
\frac{(2 j) \ldots(3 j-1)}{(j+1) \ldots(2 j-1)}>\frac{2}{3}(j-1)
$$

and since clearly

$$
\frac{(2 j) \ldots(3 j-1)}{(j+1) \ldots(2 j-1)}>\left(\frac{3}{2}\right)^{j-1},
$$

it suffices to prove that

$$
\left(\frac{3}{2}\right)^{j}>j-1, \quad j \geq 3 .
$$

This can be done by easy induction on $j$.

\section{References}

Bieniek M (2014a) Comparison of the bias of trimmed and Winsorized means, to appear in Comm. Statist, Theory Methods

Bieniek M (2014b) Optimal bounds on the bias of quasimidranges, submitted

Danielak K (2003) Sharp upper mean-variance bounds for trimmed means from restricted families. Statistics 37:305-324

Danielak K, Rychlik T (2003) Exact bounds for the bias of trimmed means. Aust N Z J Stat 45:83-96

Goroncy A (2009) Lower bounds on positive $L$-statistics. Comm Statist Theory Methods 38:1989-2002

Moriguti S (1953) A modification of Schwarz's inequality with applications to distributions. Ann Math Statistics 24:107-113

Mosteller F (2006) On some useful “inefficient” statistics. In: Hoaglin DG (ed) Selected Papers of Frederick Mosteller. Springer, New York, pp 69-100

Okolewski A, Kałuszka M (2008) Bounds for expectations of concomitants. Stat Papers 49:603-618

Raqab MZ (2007) Bounds on the bias of Winsorized means. Aust N Z J Stat 49:51-60

Rosenberger JL, Gasko M (1983) Comparing location estimators: trimmed means, medians, and trimean. In: Mosteller F, Tukey JW, Hoaglin DC (eds) Understanding robust and exploratory data analysis. Wiley, New York, pp 297-338

Rychlik T (1998) Bounds for expectations of $L$-estimates. In: Balakrishnan N, Rao CR (eds) Order statistics: theory \& methods. Handbook of statist., vol 16, North-Holland, Amsterdam, pp 105-145

Rychlik T (2001) Projecting statistical functionals, vol 160., Lecture Notes in Statistics Springer-Verlag, New York

Rychlik T (2010) Evaluations of generalized order statistics from bounded populations. Stat Papers 51:165177

Schoenberg IJ (1959) On variation diminishing approximation methods. In: Langer RE (ed) On numerical approximation. Proceedings of a Symposium, Madison, April 21-23, 1958, The University of Wisconsin Press, Madison, pp 249-274.

Tukey JW (1977) Exploratory data analysis. Addison-Wesley, Boston 